\title{
Characterization of Candidate Anti-Allergic Probiotic Strains in a Model of Th2-Skewed Human Peripheral Blood Mononuclear Cells
}

\author{
Sébastien Holvoet ${ }^{\mathrm{a}} \quad$ Adrian W. Zuercher $^{\mathrm{a}} \quad$ Françoise Julien-Javaux $^{\mathrm{b}}$ \\ Marie Perrot ${ }^{c}$ Annick Mercenier ${ }^{a}$ \\ ${ }^{a}$ Allergy Group, Department of Nutrition and Health, ${ }^{b}$ Food Safety Microbiology Group, Department of \\ Quality and Safety, and ' Applied Mathematics Group, Department of BioAnalytical Science, Nestlé Research \\ Center, Lausanne, Switzerland
}

\section{Key Words}

Allergy $\cdot$ Anti-CD $40 \cdot$ Interleukin-4 $\cdot$ Peripheral blood mononuclear cells $\cdot$ Probiotics $\cdot$ Th2 cytokines

\begin{abstract}
Background: Pre-clinical and clinical studies have evaluated the efficacy of probiotics in allergy. However, predictive in vitro systems for rational strain selection are still missing. Methods: We developed a novel in vitro screening system for the characterization of probiotics with anti-allergic potential. In this model, human peripheral blood mononuclear cells (PBMC) from healthy donors $(n=68)$ were skewed towards a Th2 cytokine phenotype by culture with IL-4 and anti-CD40, to resemble cells from allergic donors. Th2skewed cells were then co-cultured with probiotics; a total of 35 strains were tested. Levels of IFN- $\gamma$, IL-10, IL- 5 and 7 additional cytokines in culture supernatants were determined by ELISA or multiplex assay. Gene expression was assessed by real-time PCR. For validation, splenocytes from ovalbuminprimed mice and PBMC from grass-allergic donors were restimulated with respective antigen and co-cultured with probiotics, and cytokine profiles were correlated. Results: Culture with IL-4 and anti-CD40 antibody induced secretion of IL-5 from PBMC, indicative of induction of a Th2 pheno-
\end{abstract}

type. Cytokine profiles induced by probiotics were strain specific even though species- and genus-specific clustering was observed for many strains by principal component analysis. This was paralleled by mRNA levels of the corresponding genes such as increased Tbet and reduced GATA-3 gene expression. Cytokine profiles induced by probiotics in PBMC stimulated with IL-4 and anti-CD 40 correlated with those obtained from allergen-stimulated murine splenocytes or human PBMC from grass-allergic donors. Conclusions: Cytokine profiling of probiotic strains with IL-4-/anti-CD40-stimulated PBMC allowed to determine the effect of probiotics on Th2-skewed cells and thus to classify probiotic strains with anti-allergic potential.

Copyright $\odot 2013$ S. Karger AG, Basel

\section{Introduction}

The allergy pandemic nowadays affects $20-30 \%$ of the population in developed countries [1]. The spectrum of allergic symptoms is wide and ranges from atopic dermatitis (AD)/eczema in food-sensitized babies and young children to respiratory symptoms (such as allergic rhinitis and asthma) in adolescents and adults sensitized to grass and tree pollen, house dust mites and animal dan-

\section{KARGER}

Fax +4161306 1234

E-Mail karger@karger.ch

www.karger.com
(C) 2013 S. Karger AG, Basel

$1018-2438 / 13 / 1612-0142 \$ 38.00 / 0$

Accessible online at:

www.karger.com/iaa
Correspondence to: Dr. Sébastien Holvoet

Allergy Group, Department of Nutrition and Health, Nestlé Research Center Vers-chez-les-Blanc

CH-1000 Lausanne 26 (Switzerland)

E-Mail sebastien.holvoet@rdls.nestle.com 
der. Simple and cost-effective prevention strategies could help to lower the medical and economic burden associated with allergic diseases.

Clinical trials to evaluate the efficacy of probiotics in the prevention or treatment of various allergic conditions have yielded sometimes controversial results. Some studies indicated beneficial effects in the primary prevention of atopic eczema in infants [2-6] while others failed to confirm these findings [7-9] (see also meta-analysis by Lee et al. [10]). Results were generally less encouraging for treatment of $\mathrm{AD}$ [11] even though some studies showed positive results [reviewed in ref. 12-14]. Moreover, a few studies have demonstrated the efficacy of probiotics in symptom reduction of respiratory allergy in adult patients [15-17]. The interplay of numerous factors, including the environment (e.g. microbiota composition [18] and birth delivery mode), the host (e.g. genetic predisposition) and the probiotic strain itself (strain properties, dose and time of administration) are critical in determining the success of probiotic intervention against allergy [19]. Generally, for many clinically applied probiotic strains a compelling rationale for its use in a particular indication is not evident.

Thus, simple and predictive in vitro screening models could greatly facilitate rational strain selection and would enhance the chances of success in subsequent clinical trials. Determination of cytokine profiles ('immunoprofiles') induced by probiotics in human peripheral blood mononuclear cells (PBMC) $[20,21]$ or dendritic cells (DC) [22] has been applied in several studies for the characterization of probiotics. For example, an anti-inflammatory PBMC cytokine profile (low IL-12, high IL-10 along with low IFN- $\gamma$ and TNF- $\alpha$ ) was predictive of a protective anti-inflammatory effect in vivo in a mouse colitis model [23]. Cytokine profiles prognostic of an anti-allergic effect need to be established yet. One limitation to this end is the fact that normal human PBMC or CD4+ $\mathrm{T}$ cells do not secrete Th2 cytokines, which are a hallmark of an allergic state. Fujiwara et al. [24] partly overcame these limitations by using Th2-skewed splenocytes from ovalbumin (OVA)-primed mice. Notably, a strain of Lactobacillus paracasei selected on the basis of its ability to induce IL-12 and inhibit IL-4 secretion in this model showed a benefit in a subsequent clinical trial in humans suffering from allergic rhinitis [25]. Alternatively, Vissers et al. [26] recently showed that co-culture of specific probiotic strains with PBMC from human allergic donors decreased IL-13 production along with strain-specific effects on induction of Th1 cytokines and cell surface markers.

Th2-Skewed PBMC for Characterizing Probiotics
Here we report the development and use of a model of Th2-skewed human PBMC from normal donors for screening and immunological characterization of probiotic strains with anti-allergic potential. We show that strain-specific profiles were obtained and that various potentially anti-allergic immunoprofiles were found. Importantly, we show that cytokine profiles correlated well between this model and two other in vitro models based on allergen-primed Th2-skewed cells, namely OVA-specific murine splenocytes as well as PBMC from grass-allergic human donors. Linking these findings with in vivo effects may help to more rationally select probiotic strains for intervention against allergy.

\section{Materials and Methods}

\section{Reagents and Bacterial Biomass}

Bacterial biomass (kindly provided by the Food and Health Microbiology Group, Nestlé Research Center: B. Bourqui, D. de Malprade and N. Pagé) was produced by culture of each strain under optimal conditions in liquid cultures. Lactic acid bacterial strains were grown in standard growth conditions described in the literature, i.e. MRS \pm cysteine or M17 at 30,37 or $40^{\circ} \mathrm{C}$ under anaerobiosis or not, depending on the species. Escherichia coli was grown in Luria broth. Growth kinetics were determined for each strain and according to these, biomass was harvested $3 \mathrm{~h}$ after reaching the stationary phase. At this time point, cultures were washed in cold PBS and frozen in PBS $20 \%$ glycerol at $-80^{\circ} \mathrm{C}$ in $50-\mu \mathrm{l}$ aliquots. Further details on probiotic strains are given in table 1. Lipopolysaccharide (LPS) from E. coli 0111:B4 was purchased from Sigma (Buchs, Switzerland).

\section{Isolation and Culture of Th2-Skewed Human PBMC from}

Normal, Healthy Donors

Regular blood donations of healthy volunteers were collected and filtered to separate plasma from cells at the Transfusion Center of the Lausanne University Hospital (Centre Hospitalier Universitaire Vaudois). Human PBMC were isolated from these filters by flushing the cells trapped in the filters back into the blood collection bag with $90 \mathrm{ml}$ of Hanks balanced salt solution (HBSS; Sigma). The cells were diluted 1:2 with HBSS and the PBMC were isolated by density gradient centrifugation on Histopaque (Sigma). The cells at the interphase were collected and washed two times with HBSS. The PBMC were resuspended in complete Iscove's modified Dulbecco's medium (cIMDM; Sigma) containing $10 \%$ fetal bovine serum (FBS; Bioconcept, Paris, France), 1\% Lglutamine (Sigma), $1 \%$ penicillin/streptomycin (Sigma) and $0.1 \%$ gentamycin (Sigma). The cells were cultured in 48-well plates (Milian, Meyrin, Switzerland) at $1.5 \times 10^{6}$ cells $/ \mathrm{ml}$ in the presence of $50 \mathrm{ng} / \mathrm{ml}$ of IL-4 (Bioconcept) and $1 \mu \mathrm{g} / \mathrm{ml}$ of anti-CD40 antibody (R\&D Systems, Abington, UK) in cIMDM to induce a Th2 2 cytokine phenotype. LPS was used at $1 \mu \mathrm{g} / \mathrm{ml}$. After 3 days of culture, probiotics were added at $10^{7}, 10^{6}$ and $10^{5} \mathrm{CFU} / \mathrm{ml}$. After adding ingredients, PBMC culture was continued for an additional $48 \mathrm{~h}$ resulting in total culture duration of 5 days.

Int Arch Allergy Immunol 2013;161:142-154 143 
Isolation and Culture of Th2-Skewed, OVA-Primed Murine Splenocytes

Female BALB/c mice, 6-8 weeks old (Charles-River, l'Abresles, France), were sensitized intraperitoneally with $50 \mu \mathrm{g} / \mathrm{ml}$ OVA (Sigma) and aluminum sulfate (alum; $1 \mathrm{mg} / \mathrm{mouse}$; Merck, Dietikon, Switzerland) in a volume of $200 \mu \mathrm{l} /$ mouse. On day 8 , a second sensitization was carried out by the subcutaneous route with OVA and alum as described above. The mice were sacrificed on day 15 by cervical dislocation. The spleens were collected into complete RPMI 1640 (Sigma) supplemented with 10\% FBS, 1\% Lglutamine, $1 \%$ penicillin/streptomycin, $0.1 \%$ gentamycin and 50 $\mu$ M 2-mercaptoethanol (Sigma). Spleens were homogenized in cRPMI to obtain a single-cell solution. Erythrocytes were lysed with red blood cell lysis solution (Roche, Rotkreuz, Switzerland). After washing, the cells were cultured in 48 -well plates at $2 \times 10^{6}$ cells $/ \mathrm{ml}$ in the absence or presence of OVA at $1,000 \mu \mathrm{g} / \mathrm{ml}$. Probiotics were added concomitantly for a total culture duration of 5 days. Analysis of cell viability during development of the various in vitro cell culture systems confirmed that cell viability at the given concentrations of OVA and bacteria was not compromised.

\section{Isolation and Culture of PBMC from Grass-Allergic Human}

Donors

Heparinized, venous blood $(50 \mathrm{ml})$ from male and female adults (18-40 years old) with confirmed allergy (allergic rhinoconjunctivitis or asthma) to grass pollen were collected at the beginning of pollen season 24-48 h after the first occurrence of symptoms. PBMC were isolated as described above. Cells were resuspended in RPMI (Sigma) complemented with 8\% of human serum AB (Blutspendedienst SRK, Bern, Switzerland), 1\% L-glutamine, $1 \%$ penicillin/streptomycin, $1 \%$ of non-essential amino acids (Invitrogen, Lucerne, Switzerland), 1\% sodium pyruvate (Invitrogen) and $0.1 \%$ gentamycin (Sigma). Cells were cultured in 96-well plates (Milian) at $1 \times 10^{6}$ cells $/ \mathrm{ml}$ in three different conditions: (i) in medium, (ii) in the presence of $90 \mu \mathrm{g} / \mathrm{ml}$ grass allergen pollen extract (ALK-Abello wässrig 6 grass, Volketswil, Switzerland) or (iii) in the presence of $50 \mathrm{ng} / \mathrm{ml}$ of IL- 4 and $1 \mu \mathrm{g} /$ $\mathrm{ml}$ of anti-CD40. After 2 days of culture, probiotics were added at $10^{7} \mathrm{CFU} / \mathrm{ml}$. PBMC culture was continued for an additional 3 days resulting in total culture duration of 5 days.

\section{Cytokine ELISA}

Human IFN- $\gamma$, human IL-5, human IL-10, mouse IFN- $\gamma$, mouse IL-13 and mouse IL-10 were measured using DuoSet kits from $\mathrm{R} \& \mathrm{D}$ systems according to the manufacturer's instructions.

\section{Evaluation of ELISA Results}

Optical density values were transformed into pictograms per milliliter using the standard curves. To adjust for the large donorto-donor variation generally observed with human PBMC, data were standardized according to an arbitrary internal standard. The value of IFN- $\gamma(\mathrm{pg} / \mathrm{ml})$ obtained for each donor by stimulation with LPS was set to $100 \%$. For normalization of IL-10 values, the amount of IL-10 induced with strain Bifidobacterium lactis NCC 2818 (CNCM-I3446 deposited by Nestlé) was considered as 100\%. Finally, the amount of IL-5 induced with IL-4 and anti-CD40 antibody in medium only (i.e. in the absence of probiotics) was set to $100 \%$. As the effect of the different candidate probiotics was highly strain dependent, the concentration $\left(10^{5}, 10^{6}\right.$ or $\left.10^{7}\right)$ yielding optimal stimulation (for IFN- $\gamma$ and IL-10) or optimal inhibition (for IL-5) was used for the calculation of percentages as shown in table 1.

\section{Multiplex Cytokine Assay}

The concentrations of human IFN- $\gamma$, IL-10, IL-5, IL-1 $\beta$, IL-2, IL-4, IL-6, IL-8, IL-12p70, TNF- $\alpha$ and TNF- $\beta$ were measured using the human Th1/Th2 11-plex multiplex kit (Bender MedSystems, Lucerne, Switzerland) according to the manufacturer's instructions. Samples were measured on a flow cytometer (FACS Calibur; Becton Dickinson, Franklin Lakes, N.J., USA), and analyzed with FlowCytomix Pro 2.1 software (BMSFFS/2.1; Bender MedSystems).

\section{Quantitative Gene Expression Levels by Real-Time PCR}

PBMC $\left(1.5 \times 10^{6}\right.$ cells $\left./ \mathrm{ml}\right)$ were cultured in cIMDM with or without IL-4 $(50 \mathrm{ng} / \mathrm{ml})$ and anti-CD40 $(1 \mu \mathrm{g} / \mathrm{ml})$ for 3 days. Thereafter, probiotic candidates were added to PBMC and cells were collected after 10 and $24 \mathrm{~h}$. Total RNA was extracted from stimulated PBMC with the SV total RNA isolation system kit (Promega, Wallisellen, Switzerland) including a DNase treatment according to the manufacturer's instructions. Total RNA was quantified using the Ribogreen RNA quantification kit (Molecular Probes, Basel, Switzerland). Reverse transcription was performed on $1 \mu \mathrm{g}$ of total RNA by using the Multiscribe reverse transcriptase kit (Applied Biosystems, Foster City, Calif., USA). Total RNA was mixed with $50 \mu \mathrm{M}$ of random hexamers, $0.5 \mathrm{mM}$ of dNTPs, $20 \mathrm{U}$ of RNase inhibitor (Applied Biosystems), $62.5 \mathrm{U}$ of Multiscribe reverse transcriptase, $1 \times$ RT buffer and $5.5 \mathrm{~mm}$ of $\mathrm{MgCl}_{2}$ in a final volume of $100 \mu \mathrm{l}$. Human IFN- $\gamma$, IL-10, IL-5, Tbet, GATA3 and FoxP3 (Applied Biosystems) were quantified by real-time PCR (ABI PRISM 7900HT; Applied Biosystems) using the TaqMan gene expression assays. Quantification was normalized with the mean of 3 housekeeping genes: $\beta$-actin, GAPDH and HPRT (Applied Biosystems).

Based on the cycle threshold $\left(C_{t}\right)$ values obtained, a relative and normalized mRNA expression was determined for each gene using $\Delta C_{t}$. The $C_{t}$ value for each gene was corrected by the $C_{t}$ mean of the three housekeeping genes. The results were calculated as a relative expression using the formula $2^{-\Delta C_{t}} \times K$.

\section{Principal Component Analysis}

Numerical cytokine values $(\mathrm{pg} / \mathrm{ml})$ determined by multiplex cytokine assay (see above) for each strain were used to perform a principal component analysis (PCA, dimensioning technique) using R v2.14.1 Development Core Team software 2011 (R Foundation for Statistical Computing, Vienna, Austria; http://www.Rproject.org/). Data were standardized (substraction of the averages and division by the standard deviation over the individuals, the bacteria) in order to give the same weight to each variable, the cytokines.

\section{Results}

Probiotics Modulate Cytokine Production in a Model of Th2-Skewed Human PBMC

To induce a Th2 cytokine profile, human PBMC from normal, healthy donors were incubated with IL-4 and anti-CD40 antibody. This did not lead to spontaneous IFN$\gamma$ or IL-10 production, while secretion of IL-5 became detectable after 3-4 days of culture (fig. 1). Levels of IL-5 
Table 1. Cytokine profiles of Th2-skewed human PBMC stimulated with probiotics

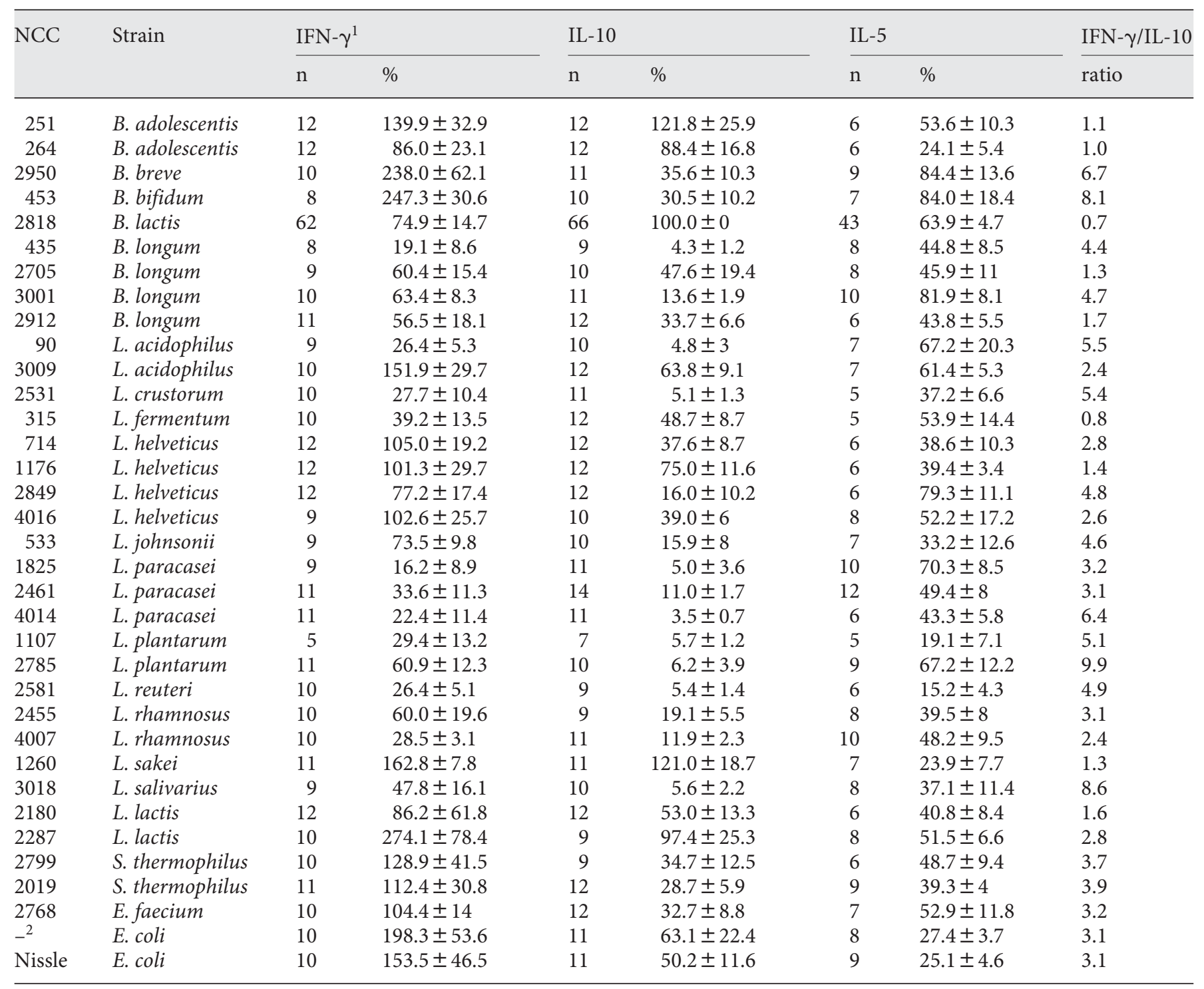

Data are expressed as medians \pm SEM. NCC = Nestlé Culture Collection; B. = Bifidobacterium; L. = Lactobacillus or Lactococcus; $S .=$ Streptococcus; E. faecium $=$ Enterococcus faecium; $n=$ number of donors.

${ }^{1}$ To equalize large donor-to-donor variations, pg/ml values were normalized to \% according to internal references as described in the Materials and Methods Section.

2 This probiotic strain of E. coli was first described in Lodinová-Zádníková et al. [40].

were highly donor dependent (fig. 1-3), including a considerable proportion of 'non-responders'. Based on these initial results, Th2-skewing of PBMC was induced for 3 days before adding probiotics. After addition of probiotic candidates or LPS, culture was continued for 2 more days, thus leading to a total culture period of 5 days. As shown in figure $2 \mathrm{a}$ for a single donor, co-culture with probiotics according to this protocol led to secretion of IFN- $\gamma$ and
IL-10, and to inhibition of IL-5 secretion in a strain-specific fashion. Interestingly, IL-4/anti-CD40 conditioning rendered cells more reactive to IFN- $\gamma$ secretion upon addition of probiotics (grey bars) compared to unconditioned (medium; white bars) PBMC (fig. 2a, left). In contrast, capability of IL-10 secretion was reduced in Th2skewed cells compared to PBMC cultured in medium alone (fig. $2 \mathrm{a}$, middle). As shown before, IL-5 production 
Fig. 1. Characteristics of a model of Th2skewed human PBMC. Human PBMC were cultured in medium only $(\mathbf{a}-\mathbf{c})$ or in medium with IL-4 and anti-CD40 antibody (d-f). Kinetics of cytokine production (measured by ELISA) for 9 randomly chosen donors are shown.

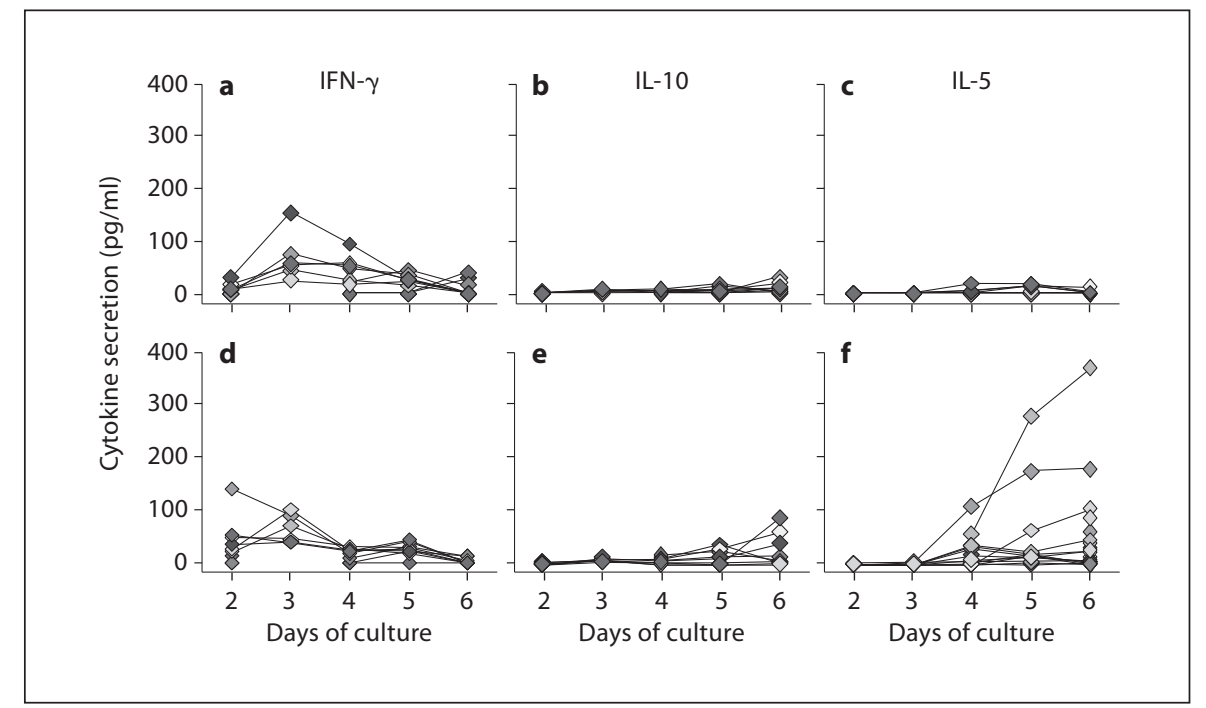

was a unique feature of IL-4/anti-CD40-conditioned PBMC (fig. 2a, right). Induction of IFN- $\gamma$ and IL-10 as well as inhibition of IL-5 secretion was dependent on the dose of probiotics, as shown for 5 individual strains (fig. 2b).

\section{Immunoprofiling of Probiotic Bacterial Strains on \\ Th2-Skewed Human PBMC}

PBMC from a total of 68 donors were used in this study and results of 61 (IFN- $\gamma$ ), 65 (IL-10) and 45 (IL-5) donors could be evaluated; the number of IL- 5 data sets was lower due to several non-responders, i.e. cells from donors producing no or only minute amounts of IL-5. As shown in figure 3 , there were large interindividual differences in the absolute amounts of cytokines induced. To allow direct comparison and pooling of data obtained from different donors, results were normalized according to internal standards used in each experiment: IFN$\gamma$ levels obtained from each donor by LPS stimulation were set to $100 \%$, IL-10 was normalized according to levels induced by $B$. lactis NCC 2818 (=100\%) and IL-5 levels induced in the absence of probiotics (medium) were set to $100 \%$.

Immunoprofiles of 35 strains (9 bifidobacteria, 19 lactobacilli, 2 lactococci, 2 streptococci, 1 enterococcus and 2 E. coli) are shown in table 1 . Large variability in the induced levels of IFN- $\gamma$ and IL-10 were observed between the strains. Nevertheless, bifidobacteria were overall more robust IL-10 inducers than lactobacilli (median of 35.6 vs. 11.9\%). The 2 strains of Lactococcus, particularly $L$. lactis NCC 2287, were robust inducers of IFN- $\gamma$ and
IL-10 whereas the 2 strains of $E$. coli were efficient inducers of IFN- $\gamma$. Likewise, the efficacy of reducing IL- 5 secretion (\% residual production of IL-5 is shown) varied greatly between the strains. Nevertheless, while some rules applying to genera or species were identified, often exceptions to the rules were found, underlining the strain specificity of the candidate probiotics. The cytokine protein profiles for IFN- $\gamma$, IL-10 and IL- 5 were confirmed on gene expression levels by real-time PCR (fig. 4a). In addition, expression of Tbet, FoxP3 and GATA-3 was quantified. A slight increase in Tbet was observed, whereas FoxP3 and GATA-3 tended to be reduced (fig. 4b). However, generally the differences between strains were less marked on the level of these master transcription factors compared to the corresponding prototypic cytokines.

For a short list of strains, some were selected based on their ability of inhibiting IL-5 production, others on the basis of their documented anti-allergic effect in humans (see Discussion); a broader panel of cytokines was determined by multiplex assay (table 2). PCA revealed that based on the profile of 10 cytokines a certain level of clustering by genus was observed (fig. 5). The profiles of lactobacilli and bifidobacteria overlapped partially, whereas lactococci had a distinct profile, probably driven by their strong IL-12 production (table 2).

Correlation of Cytokine Profiles between Th2-Skewed Human PBMC and OVA-Specific Murine Splenocytes and Grass-Allergen-Specific Human PBMC

To determine whether comparable cytokine profiles were induced by probiotics in artificially Th2-skewed 


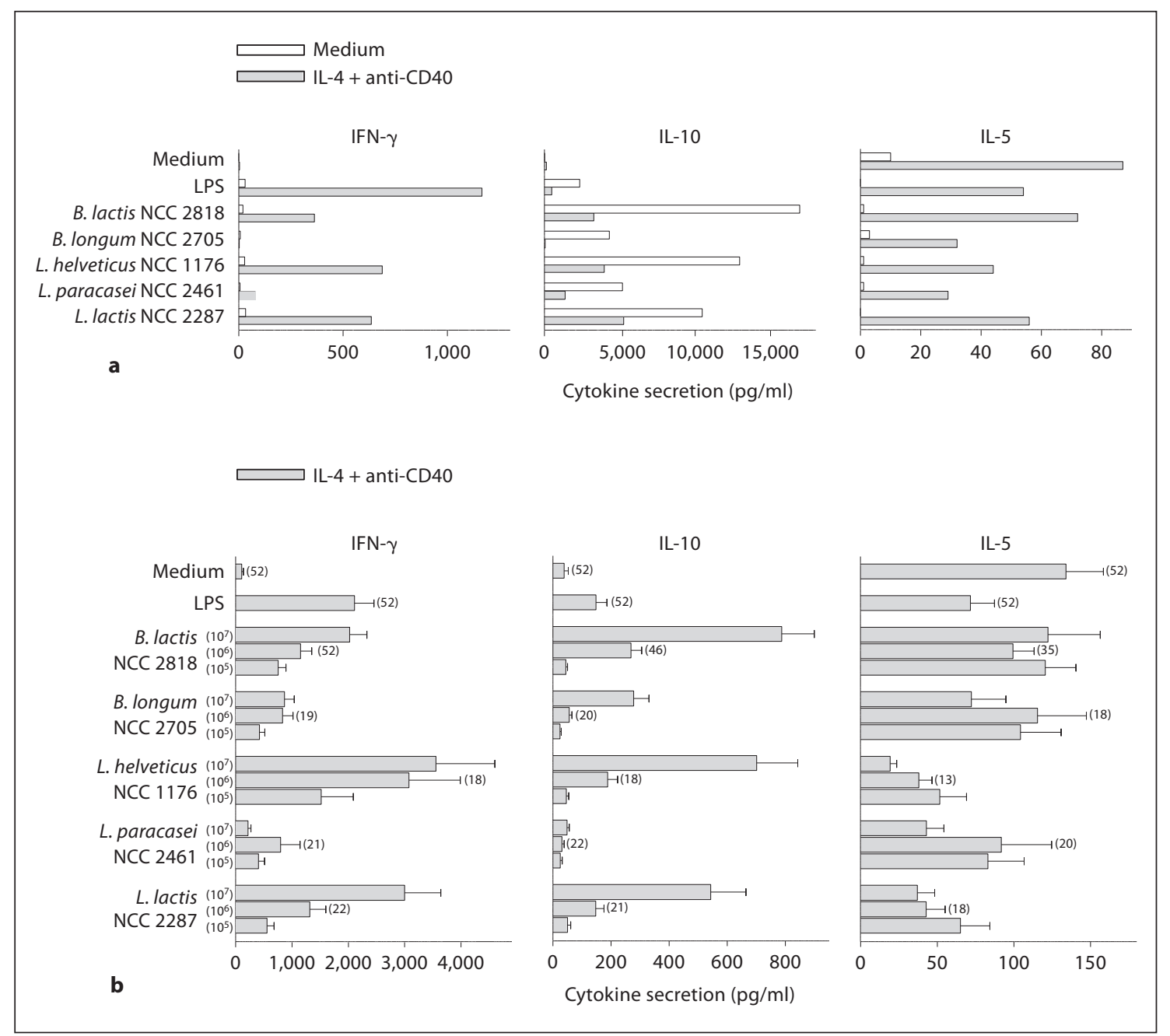

Fig. 2. Dose-dependent modulation of cytokine production by probiotics. a Modulation of cytokine production by probiotics $\left(10^{7} \mathrm{CFU} / \mathrm{ml}\right)$ in Th2-skewed vs. non-skewed (medium) cells. Data from 1 representative donor are shown. b Human PBMC cultured in the presence of IL-4 and anti-CD40 antibody were stimulated with probiotic strains at various doses. Cytokines were determined by ELISA. Data (mean \pm SEM) from 13-55 donors (numbers of donors in parentheses) are shown (depending on the strain and cytokine).

PBMC versus allergen-stimulated cells, a selection of strains (again mainly chosen based on their ability of inhibiting IL-5 production) was tested on OVA-specific murine splenocytes [24] and on PBMC collected from grass-allergic donors at the beginning of the pollen season and restimulated with grass pollen. Strong correlation of IFN- $\gamma$ and IL-10 levels between Th2-skewed PBMC and OVA-specific splenocytes (fig. 6a) was observed for most but not all strains (non-correlating strains excluded post hoc are shown in white). Similarly, levels of murine IL-13 correlated with human IL-5 induced with IL-4/anti-CD40. Profiles obtained from grass-allergen- specific PBMC (fig. 6b) correlated with Th2-skewed PBMC for IFN- $\gamma$ and IL-10. In contrast, no correlation of IL-5 levels was observed, which was most likely due to the fact that strains were chosen based on their ability to inhibit IL-5 secretion, resulting in low variability in this parameter. Taken together, these data demonstrate that similar cytokine profiles were induced by individual probiotics in artificially Th2-skewed PBMC in the absence of allergen and 'naturally' Th2-skewed PBMC or splenocytes under allergen-specific restimulation. These findings validate the use of IL-4-/anti-CD40-stimulated cells to mimic allergen-specific cells. 
Fig. 3. Range of cytokine production by all donors included in the study: IFN- $\gamma$ induced by $E$. coli LPS, IL-10 induced by probiotic strain B. lactis NCC 2818 and IL-5 production induced by IL-4 + anti-CD40 without addition of probiotics. Cytokines were measured by ELISA. These stimuli were included in all experiments to allow normalization of values between donors as described in the text. Grey diamonds represent individual donors (medians \pm interquatile ranges).

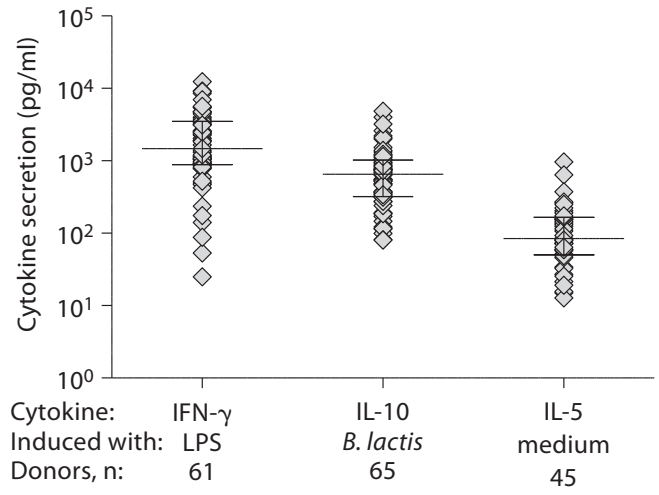

IL-10
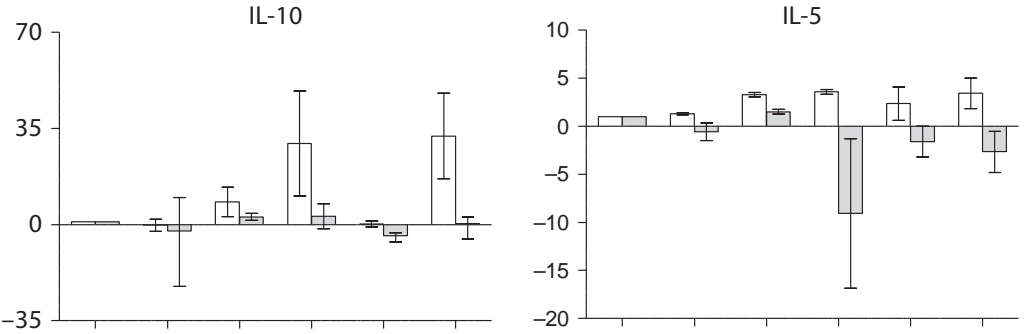

FoxP3
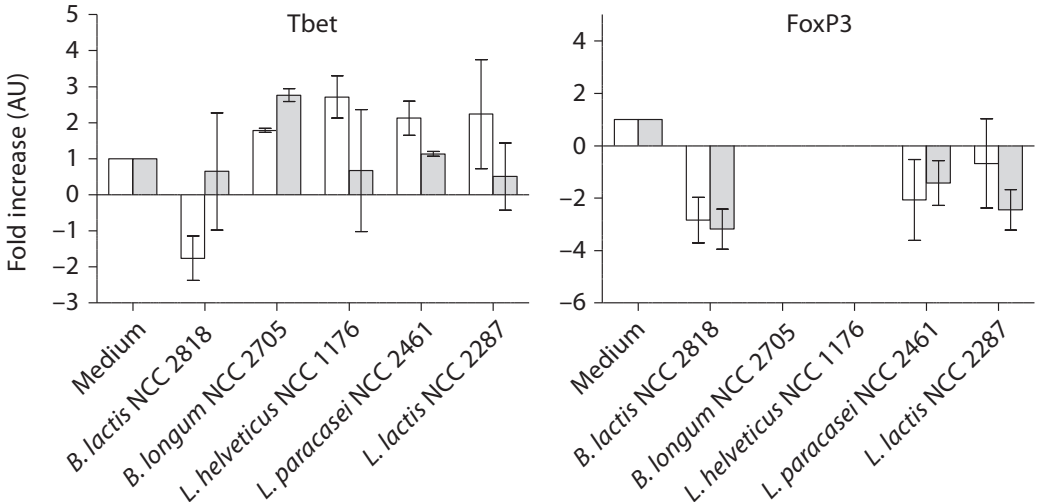

Fig. 4. Gene-expression of Th2-skewed human PBMC stimulated with probiotics. Human PBMC cultured in medium with IL-4 and anti-CD40 antibody were stimulated with probiotic strains at optimal doses. Cells were sampled 10 and $24 \mathrm{~h}$ after addition of probiotics, and gene expression levels were quantified by RT-PCR.
Relative gene expression (in arbitrary units, AU) was normalized to 3 housekeeping genes. Data are means \pm SEM from 2 donors for NCC 2818, NCC 2705 and NCC 1176, and 4 donors for NCC 2461 and NCC 2287. 


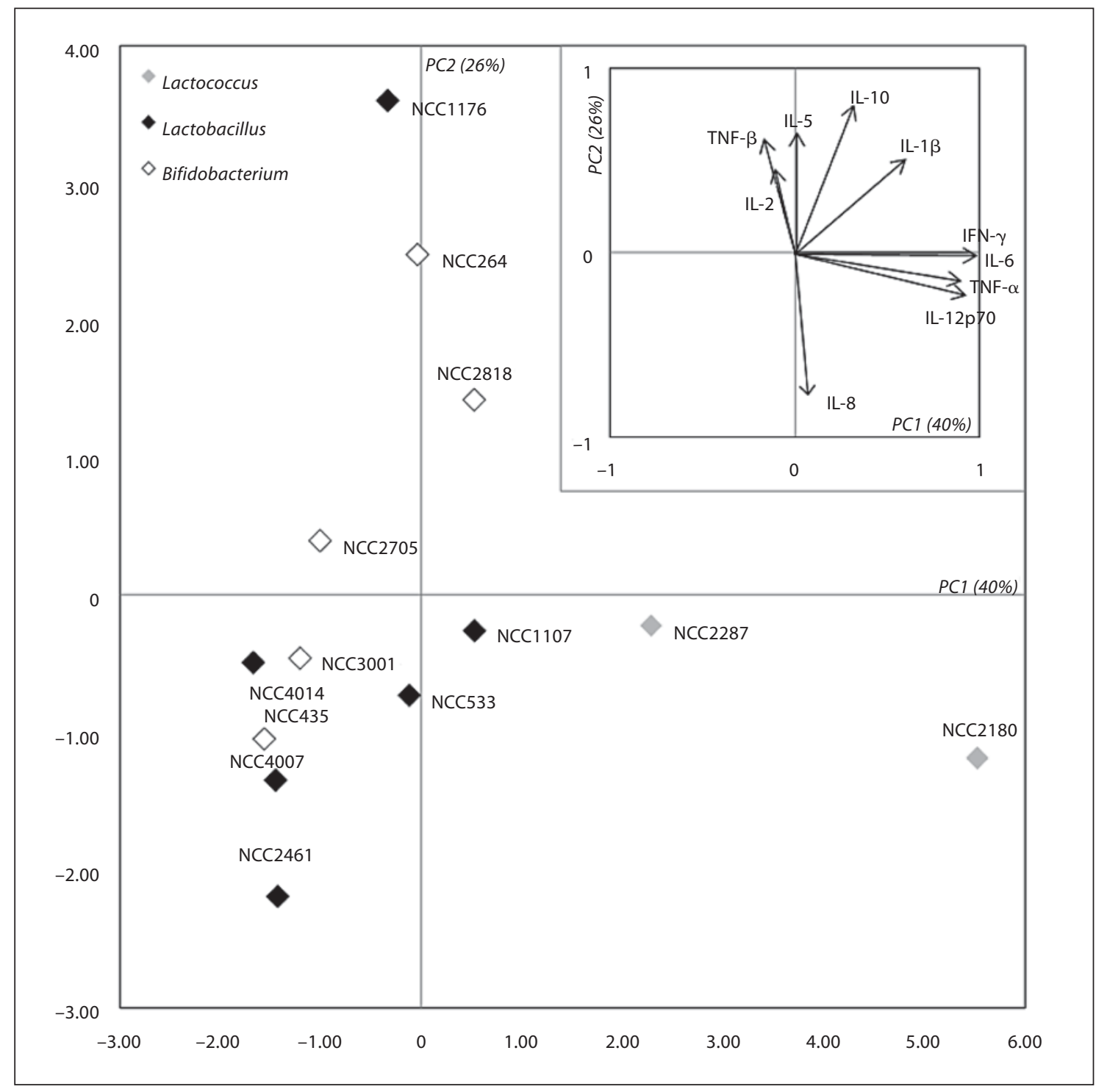

Fig. 5. PCA of 10 cytokines produced by Th2-skewed human PBMC stimulated with probiotics. Human PBMC cultured in medium with IL-4 and anti-CD40 antibody were stimulated with probiotics. Ten cytokines were determined by multiplex assay. Medians of cytokines were calculated for each strain on 7 donors. The bi-plot shows the first 2 principal components of the PCA (PC1 and PC2). Bacteria are represented on these 2 axes, and an- other plot (top right) shows the directions of each variable (cytokines) on these same axes. The aim of this plot is to provide a visual representation of the data, in only 2 dimensions, to summarize the information $(40+26=66 \%$ of the variance of the data is explained on this plot). Details on NCC codes shown in the figure are described in table 1.

\section{Discussion}

In this paper, we describe an in vitro model for immunological characterization of probiotics for anti-allergic intervention. It allowed us to determine unique cytokine profiles for probiotic strains of different genera and species. Particularly, it permitted to address the ability of dif- ferent strains to interfere with Th2 cytokine (IL-5) production by PBMC.

It has been shown previously that culture of PBMC with IL-4 and anti-CD40 leads to Th2-skewing and eventually to IgE antibody production in cell culture supernatant [27]. On the other hand, co-culture of PBMC with bacteria has frequently been used for characterization of 
Table 2. Cytokines by Th2-skewed human PBMC stimulated with probiotics (multiplex analysis) ${ }^{1}$

\begin{tabular}{|c|c|c|c|c|c|c|c|c|c|c|c|}
\hline NCC & Strain & IL-12p70 & IFN- $\gamma$ & IL-2 & IL-10 & IL-8 & IL-6 & IL-5 & $L-1 \beta$ & TNF- $\alpha$ & TNF- $\beta$ \\
\hline & LPS & $20 \pm 11$ & $582 \pm 281$ & $40 \pm 11$ & $88 \pm 21$ & $2,897 \pm 1,813$ & $163 \pm 2,176$ & $13 \pm 44$ & $4 \pm 5$ & $80 \pm 273$ & $147 \pm 97^{* *}$ \\
\hline 2818 & B. lactis & $20 \pm 61$ & $637 \pm 178$ & $61 \pm 36$ & $902 \pm 183^{*}$ & $3,562 \pm 1,130$ & $10,255 \pm 3,665^{*}$ & $20 \pm 110$ & $155 \pm 585^{*}$ & $1,243 \pm 4,893$ & $212 \pm 319^{* *}$ \\
\hline 001 & B. longum & 292 & 102 & $53 \pm 39$ & $9 \pm 35^{*}$ & $9,768 \pm 2,048$ & $2,683 \pm 3,113^{*}$ & $22 \pm 105$ & $20 \pm 508^{*}$ & $618.2 \pm 4,583$ & $298 \pm 170^{* *}$ \\
\hline 2705 & B. longum & \pm 137 & $205 \pm 200$ & $41 \pm 35$ & $225 \pm 92^{*}$ & $4,751 \pm 1,531$ & $3,501 \pm 3,409^{*}$ & $14 \pm 110$ & $94 \pm 396^{*}$ & $927 \pm 5,431$ & $333 \pm 159^{* * *}$ \\
\hline 1176 & L. helveticus & \pm 366 & $69 \pm 415$ & $69 \pm 39$ & $741 \pm 319^{*}$ & $3,073 \pm 1,169$ & $4,952 \pm 3,743^{*}$ & $27 \pm 85$ & $156 \pm 481^{*}$ & $1,609 \pm 5,146$ & $366 \pm 264^{* *}$ \\
\hline 533 & L. johnsonii & $585 \pm 527$ & $704 \pm 121$ & $51 \pm 45$ & $306 \pm 71^{*}$ & $11,161 \pm 2,055$ & $64,104 \pm 2,952^{*}$ & $18 \pm 75$ & $28 \pm 231^{*}$ & $974 \pm 3,819$ & $279 \pm 262^{* * *}$ \\
\hline 4014 & L. paracasei & $125 \pm 287$ & $68 \pm 84$ & $40 \pm 22$ & $35 \pm 22^{*}$ & $6,275 \pm 1,420$ & $1,452 \pm 1,450^{*}$ & $18 \pm 91$ & $5 \pm 219^{*}$ & $134 \pm 3,875$ & $306 \pm 126^{* *}$ \\
\hline 2461 & L. paracasei & 49 & $137 \pm 78$ & $44 \pm 24$ & $62 \pm 20^{*}$ & $11,584 \pm 2,521$ & $805 \pm 1,269^{*}$ & $9 \pm 68$ & $8.2 \pm 56^{*}$ & $324 \pm 3,451$ & $175 \pm 164^{* *}$ \\
\hline 1107 & L. plantarum & $339 \pm 278$ & $310 \pm 264$ & $40 \pm 14$ & $169 \pm 963^{*}$ & $6,039 \pm 1,749$ & $11,328 \pm 2,883^{*}$ & $13 \pm 85$ & $1,512 \pm 84^{*}$ & $2,448 \pm 2,439$ & $188 \pm 66^{* *}$ \\
\hline 4007 & L. rhamnosus & $49 \pm 55^{*}$ & $69 \pm 77^{*}$ & $16 \pm 31^{*}$ & $53 \pm 19^{*}$ & $6,406 \pm 2,921^{*}$ & $691 \pm 1,016^{*}$ & $18 \pm 76^{*}$ & $12 \pm 49^{*}$ & $906 \pm 5,957^{*}$ & $218 \pm 116^{* *}$ \\
\hline
\end{tabular}

Data are expressed as pg/ml or medians \pm SEM. NCC $=$ Nestlé Culture Collection; B. = Bifidobacterium; L. = Lactobacillus or Lactococcus.

${ }^{1}$ Seven donors were tested, for some cytokines only $5\left({ }^{*}\right)$ or $3\left({ }^{* *}\right)$ data sets could be evaluated.

probiotics and other bacteria. A limitation of these systems when used for identification of potentially anti-allergy probiotics is the absence of spontaneous Th2-cytokine production by normal human PBMC. An alternative to overcome this limitation is the use of allergen-specific cells from laboratory animals [19] or humans [26]. While allergen-specific, Th2-skewed cells from laboratory animals are readily available, results obtained with them might not be representative for humans. In contrast, human allergen-specific PBMC can be obtained as well [26], but this includes logistic hurdles, such as restrictions for optimal bleeding time points and large donor-to-donor variability due to differences in the degree of sensitization and specificity for various allergens. Particularly for screening purposes such limitations are certainly relevant, e.g. for the characterization of the 35 strains described in this paper, a total of 68 donors were needed. Therefore, we chose artificial skewing with IL-4/anti$\mathrm{CD} 40$ to assure a more readily available and reproducible source of PBMC secreting an 'allergy-like' Th2 cytokine profile.

Our data show that similar cytokine profiles were obtained by co-culture of probiotics with IL-4-/antiCD40-conditioned PBMC versus allergen-specific human PBMC or allergen-specific murine splenocytes (fig. 6). Even though the three models operate under different conditions (e.g. culture time or dosing) and thus cannot be directly compared, establishing this correla- tion was critical for the validation of the artificial, nonantigen-specific model we used for screening. The high correlations of IFN- $\gamma$ and IL-10 for most of the strains deliver this proof. Likewise, a good correlation was observed between IL-5 from Th2-skewed human PBMC and IL-13 from OVA-specific splenocytes. In contrast, no correlation existed between IL-5 from Th2-skewed and grass-allergen-specific human PBMC. This lack of correlation is likely explained by the choice of bacterial strains for this sub-analysis: based on the first screening (table 1) only strains with a strong capability of IL-5 inhibition were further analyzed. This led to the clustering of results at approximately $200 \mathrm{ng} / \mathrm{ml}$ for IL-4-/antiCD40-stimulated IL-5 and 100-200 ng/ml for grass-allergen-induced IL-5 (fig. 6). In these narrow boundaries, small variations may lead to large reductions in correlations. Importantly, our data confirm very recent results by another laboratory showing that PBMC from allergic donors upon restimulation with anti-CD3/anti-CD28 antibody or with allergen secreted IL-13, which could be inhibited by co-culture with probiotics [26].

Comparison of cytokine profiles in IL-4/anti-CD40 versus unconditioned cells showed increased IFN- $\gamma$ and reduced IL-10 reactivity by Th2-skewed cells after probiotic stimulation (fig. 2a). It may be speculated that induction with anti-CD40 antibody led to a generally higher activation state of CD4+ T cells in PBMC which may result in a higher IFN- $\gamma$ response upon exposure to probi- 


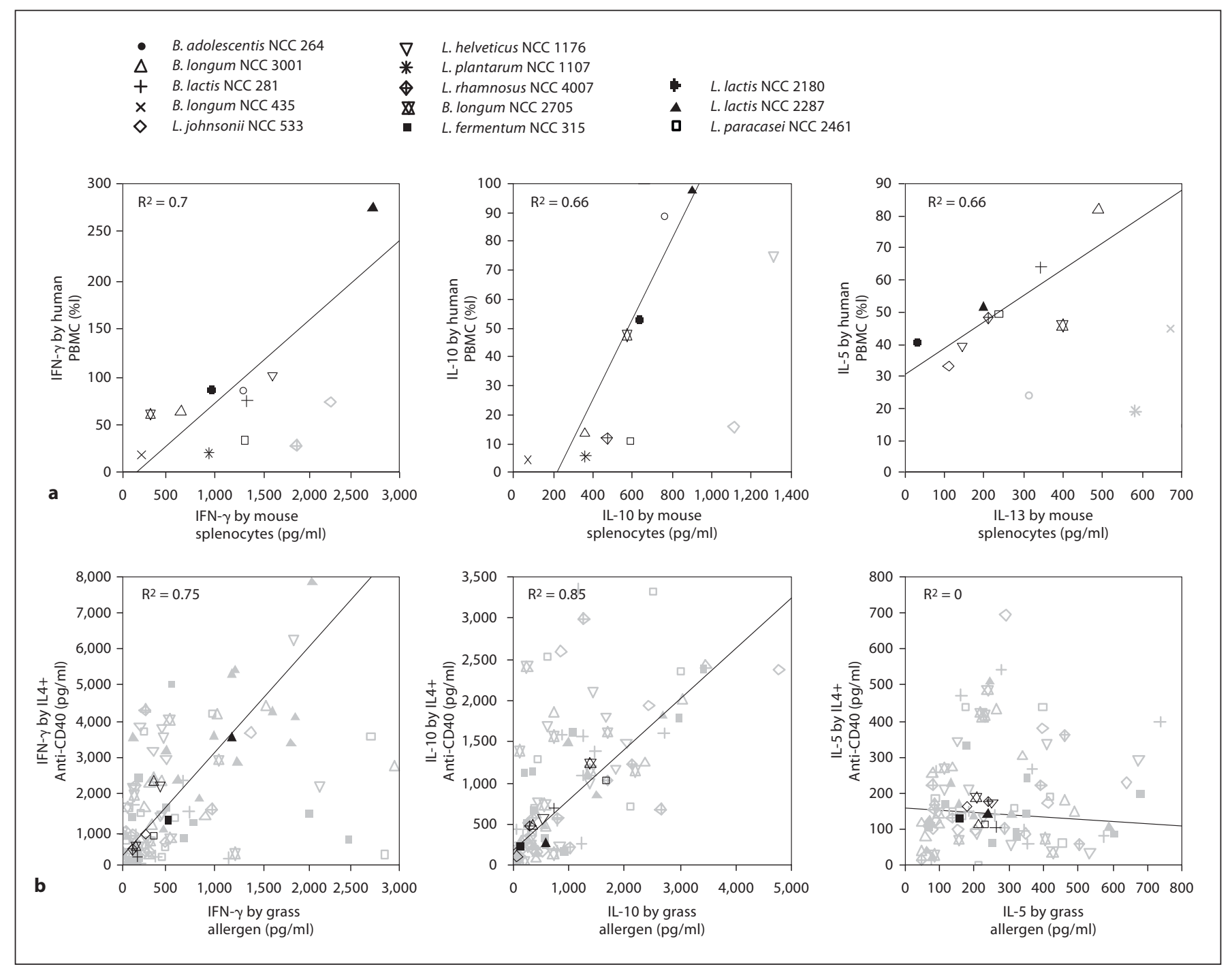

Fig. 6. Correlation of cytokine expression after probiotic stimulation of Th2-skewed human PBMC vs. allergen-specific human PBMC and murine splenocytes. Normal human PBMC were Th2 skewed with IL-4 and anti-CD40 antibody. a Mice were primed with OVA, splenocytes were restimulated with OVA. Data from 2 independent mouse experiments are included; number of human

otics. However, it has to be noted that IL-4/anti-CD40 conditioning did not lead to spontaneous IFN- $\gamma$ production (fig. 1). Reduced production of IL-10 by IL- 4 -treated cells has been described previously [28]. Yao et al. showed that treatment of DC with IL- 4 in the presence of LPS or CpG reduced IL-10 production and thus promoted Th1 differentiation of $\mathrm{CD} 4+\mathrm{T}$ cells. The differences in reactivity of allergic versus non-allergic cells have also been described in vivo. Dunstan et al. [29] showed that PBMC from patients with $\mathrm{AD}$ produced lower amounts of IFN-
PBMC donors for each strain and cytokine are shown in table 1. b PBMC from human allergic donors were restimulated with grass allergen or IL- 4 and anti-CD40 antibody. All cells were cocultured with probiotics; cytokines were measured by ELISA. Data from 4-13 donors are included (depending on the cytokine and strain).

$\gamma$, IL-10 or TNF- $\alpha$ in response to polyclonal or antigenspecific stimuli than cells from healthy counterparts. Similarly, a cross-over study comparing a mix of $L$. paracasei Lpc-37, L. acidophilus 74-2 and Bifidobacterium animalis subsp. lactis DGCC 420 (B. lactis 420) to placebo revealed differences in reactivity of PBMC from healthy subjects compared to patients suffering from $\mathrm{AD}$ [30]. However, clearly the unique feature of the model presented here is the induction of IL-5 secretion allowing the study of the effect of individual probiotic strains on Th2 
cytokine secretion in vitro. Indeed, the ability of re-directing a Th2 state may be one of the critical components for achieving primary prevention of allergy.

Comparison of the cytokine profiles stimulated by the studied strains revealed some inter-genus and inter-species differences. For example, overall bifidobacteria were more robust inducers of IL-10 compared to lactobacilli, which is reflected in the lower IFN- $\gamma / \mathrm{IL}-10$ ratio. Similarly, some recurring traits of particular species were observed, such as robust induction of IFN- $\gamma$ by (most) $L$. acidophilus and L. helveticus strains (tables 1, 2). However, often cytokine profiles were strain specific, for example revealing 2 strains of Bifidobacterium longum (NCC 435 and NCC 3001) with the capacity of inducing relatively high amounts of IFN- $\gamma$ along with low IL-10, resulting in a high IFN- $\gamma /$ IL-10 ratio while 2 other strains of B. longum (NCC 2705 and NCC 2912) had a low IFN$\gamma /$ IL-10 ratio. Taken together these data strongly underline the strain specificity of probiotics in their immunomodulatory capacity, including differences in their ability to potentially counterbalance an established Th2 cytokine profile. They also emphasize the need for screening and characterization of strains before proceeding to in vivo preclinical models or clinical studies in humans.

In contrast to the large variations between IFN- $\gamma$ and IL-10 protein and mRNA levels induced by different probiotics strains (fig. 4a), the differences between the mRNA levels for Tbet, FoxP3 and GATA-3 were minor (fig. 4b). This indicates that for immunological strain characterization in vitro determination of individual cytokines (or other yet to be defined markers) is currently a good way forward and that measurement of master transcription factors determining Th1 (Tbet), Treg (FoxP3) or Th2 (GATA-3) orientations are not sensitive enough in our assay for classification of probiotic strains. It has been suggested that Treg induction might be one of the protective mechanisms of probiotics [reviewed in ref. 31]. Lactobacillus signalling through DC-SIGN effectively induced DC that were able to drive Treg differentiation of CD4+ $\mathrm{T}$ cells [32]. Our analyses did not indicate induction of FoxP3 expression by probiotics in PBMC. Moreover, it has been shown that induction of FoxP3 in vivo was actually positively associated with AD development. This implies that FoxP3 expression is not necessarily correlated to protection, but may rather illustrate the bodies' attempt to counteract allergic sensitization/inflammation. In addition, PBMC might not be the optimal cellular source for inducing Treg.

To valorize the in vitro characterization model we need to learn how to translate the in vitro cytokine pro- files into in vivo effects and thus to determine the predictive value of the cytokine profiles, i.e. we need to determine a 'correlate of protection' for allergy. Thus, which in vitro cytokine profile may predict protection in vivo? A study for prevention of allergy showed that neonates born to mothers that had consumed Lactobacillus rhamnosus DR20 during pregnancy developed less AD in the first 2 years of life and that these infants had increased cord blood and serum IFN- $\gamma$ levels [33]. Likewise, in two intervention studies for treatment of established AD it was shown that positive clinical outcomes were associated with mild inflammatory reactions induced by the probiotic intervention. Intervention with L. rhamnosus LGG resulted in increased serum levels of CRP and IL-6 [34] and similar results were obtained with a mixture of 4 probiotics [35]. In addition, successful intervention with Lactobacillus fermentum PCC in children suffering from AD was accompanied by increased IFN- $\gamma$ and TNF- $\alpha$ production by PBMC in response to polyclonal stimuli [36]. Finally, a recent study by Snel et al. [37] showed that after consuming Lactobacillus plantarum CBS125632 for 4 weeks, PBMC from allergic donors produced less IL-5 and IL-13 but more IL-10. Collectively, these findings suggest that induction of mild inflammation and particularly of IFN- $\gamma$ secretion could be characteristic of a protective anti-allergic response, a hypothesis that has been put forward previously [38]. In our present study, we included strains which had proven anti-allergic activity in vivo either in human or in pre-clinical animal studies, for example L. rhamnosus NCC 4007 [2], which is indistinguishable by molecular typing from L. rhamnosus ATCC 53103, L. paracasei NCC 4014 which is equivalent to strain KW3110 [25], B. longum NCC 3001 which corresponds to strain BB536 [15], or L. paracasei NCC 2461 who was recently shown to reduce symptoms of allergic rhinitis [39]. Interestingly, most of these strains shared a 'discrete' cytokine profile, with low IFN- $\gamma$ and IL-10 but efficient inhibition of IL-5 production (table 1, fig. 5). Thus, a 'low-key' cytokine pattern (possibly associated with a 'homeostatic' effect conferred by the probiotic strain) might be an alternative cytokine profile with promise for clinical success. Overall, these considerations indicate that not one single but several cytokine profiles from PBMC might be predictive of protection in vivo. Evidently, anti-allergic effects of probiotics may not solely be mediated via immunomodulation, e.g. direct re-enforcement of the intestinal barrier may translate in an anti-allergic effect as well. To identify such non-immune characteristics, cytokine profiling will not be the tool of choice. 
In summary, we established a simple in vitro assay that allowed immune classification of a selection of candidate probiotic strains. This tool will be instrumental in selecting a small number of potential anti-allergic strains for further evaluation in more sophisticated in vitro models such as DC/T-cell assays and in vivo allergy models. We hypothesize that careful in vitro and in vivo characterization of probiotics will facilitate the design of clinical studies and will help to explain contradictory results observed in human studies in the area of probiotics and allergy.

\section{Acknowledgments}

We thank Bertrand Bourqui, Dominique De Malprade and Nicolas Pagé for harmonization of bacterial biomass. We thank Marietta Weiss and Audrey Demont for technical help, and Dr. Maurice Beaumont and Sylviane Oguey-Araymon for the clinical coordination. We greatly appreciate the helpful discussions and critical inputs from Guenolée Prioult, Anurag Singh, Laurence van Overtfeldt (Stallergènes SA, Paris, France) and Philippe Moingeon (Stallergènes). This project has been funded by NESTEC SA.

\section{Disclosure Statement}

The authors have no conflicts of interest.

\section{References}

1 Worldwide variation in prevalence of symptoms of asthma, allergic rhinoconjunctivitis, and atopic eczema: ISAAC. The International Study of Asthma and Allergies in Childhood (ISAAC) Steering Committee. Lancet 1998;351:1225-1232.

-2 Kalliomaki M, Salminen S, Arvilommi H, Kero P, Koskinen P, Isolauri E: Probiotics in primary prevention of atopic disease: a randomised placebo-controlled trial. Lancet 2001;357:1076-1079.

3 Kukkonen K, Savilahti E, Haahtela T, et al: Probiotics and prebiotic galacto-oligosaccharides in the prevention of allergic diseases: a randomized, double-blind, placebocontrolled trial. J Allergy Clin Immunol 2007;119:192-198.

4 Abrahamsson TR, Jakobsson T, Bottcher MF, et al: Probiotics in prevention of IgE-associated eczema: a double-blind, randomized, placebo-controlled trial. J Allergy Clin Immunol 2007;119:1174-1180.

5 Kim JY, Kwon JH, Ahn SH, et al: Effect of probiotic mix (Bifidobacterium bifidum, Bifidobacterium lactis, Lactobacillus acidophilus) in the primary prevention of eczema: a double-blind, randomized, placebo-controlled trial. Pediatr Allergy Immunol 2010; 21:e386-e393.

6 Niers L, Martin R, Rijkers G, et al: The effects of selected probiotic strains on the development of eczema (the PandA study). Allergy 2009;64:1349-1358.

7 Taylor AL, Dunstan JA, Prescott SL: Probiotic supplementation for the first 6 months of life fails to reduce the risk of atopic dermatitis and increases the risk of allergen sensitization in high-risk children: a randomized controlled trial. J Allergy Clin Immunol 2007;119:184-191.
-8 Kopp MV, Hennemuth I, Heinzmann A, Urbanek R: Randomized, double-blind, placebo-controlled trial of probiotics for primary prevention: no clinical effects of Lactobacillus GG supplementation. Pediatrics 2008; 121:e850-e856.

$\checkmark 9$ Soh SE, Aw M, Gerez I, et al: Probiotic supplementation in the first 6 months of life in at risk Asian infants - effects on eczema and atopic sensitization at the age of 1 year. Clin Exp Allergy 2009;39:571-578.

10 Lee J, Seto D, Bielory L: Meta-analysis of clinical trials of probiotics for prevention and treatment of pediatric atopic dermatitis. J Allergy Clin Immunol 2008;121:116-121.

-11 Viljanen M, Savilahti E, Haahtela T, et al: Probiotics in the treatment of atopic eczema/ dermatitis syndrome in infants: a doubleblind placebo-controlled trial. Allergy 2005; 60:494-500.

12 Weston S, Halbert A, Richmond P, Prescott SL: Effects of probiotics on atopic dermatitis: a randomised controlled trial. Arch Dis Child 2005;90:892-897.

13 Boyle RJ, Tang ML: The role of probiotics in the management of allergic disease. Clin Exp Allergy 2006;36:568-576.

14 Gerasimov SV, Vasjuta VV, Myhovych OO, Bondarchuk LI: Probiotic supplement reduces atopic dermatitis in preschool children: a randomized, double-blind, placebo-controlled, clinical trial. Am J Clin Dermatol 2010;11:351-361.

15 Xiao JZ, Kondo S, Yanagisawa N, et al: Probiotics in the treatment of Japanese cedar pollinosis: a double-blind placebo-controlled trial. Clin Exp Allergy 2006;36:14251435.

16 Peng GC, Hsu CH: The efficacy and safety of heat-killed Lactobacillus paracasei for treatment of perennial allergic rhinitis induced by house-dust mite. Pediatr Allergy Immunol 2005; 16:433-438.
17 Chen YS, Jan RL, Lin YL, Chen HH, Wang JY: Randomized placebo-controlled trial of lactobacillus on asthmatic children with allergic rhinitis. Pediatr Pulmonol 2010;45: 1111-1120.

18 Penders J, Stobberingh EE, van den Brandt PA, Thijs C: The role of the intestinal microbiota in the development of atopic disorders. Allergy 2007;62:1223-1236.

19 Prescott SL, Bjorksten B: Probiotics for the prevention or treatment of allergic diseases. J Allergy Clin Immunol 2007;120:255-262.

20 Kekkonen RA, Kajasto E, Miettinen M, Veckman V, Korpela R, Julkunen I: Probiotic Leuconostoc mesenteroides ssp. cremoris and Streptococcus thermophilus induce IL12 and IFN-gamma production. World J Gastroenterol 2008;14:1192-1203.

21 Niers LE, Timmerman HM, Rijkers GT, et al: Identification of strong interleukin-10 inducing lactic acid bacteria which down-regulate Thelper type 2 cytokines. Clin Exp Allergy 2005;35:1481-1489.

22 Mohamadzadeh M, Olson S, Kalina WV, et al: Lactobacilli activate human dendritic cells that skew T cells toward T helper 1 polarization. Proc Natl Acad Sci USA 2005; 102 : 2880-2885.

23 Foligne B, Nutten S, Grangette C, et al: Correlation between in vitro and in vivo immunomodulatory properties of lactic acid bacteria. World J Gastroenterol 2007;13:236243.

24 Fujiwara D, Inoue S, Wakabayashi H, Fujii T: The anti-allergic effects of lactic acid bacteria are strain dependent and mediated by effects on both Th1/Th2 cytokine expression and balance. Int Arch Allergy Immunol 2004;135:205-215. 
-25 Fujiwara D, Wakabayashi H, Watanabe H, Nishida S, Iino H: A double-blind trial of Lactobacillus paracasei strain KW3110 administration for immunregulation in patients with pollen allergy. Allergol Int 2005; 54:143-149.

26 Vissers YM, Snel J, Zuurendonk PF, Kleerebezem M, Wichers HJ, Savelkoul HF: Lactobacillus strains differentially modulate cytokine production by hPBMC from pollenallergic patients. FEMS Immunol Med Microbiol 2011;61:28-40.

27 Rousset F, Garcia E, Banchereau J: Cytokineinduced proliferation and immunoglobulin production of human B lymphocytes triggered through their CD40 antigen. J Exp Med 1991;173:705-710.

28 Yao Y, Li W, Kaplan MH, Chang CH: Interleukin (IL)-4 inhibits IL-10 to promote IL-12 production by dendritic cells. J Exp Med 2005;201:1899-1903.

29 Dunstan JA, Hale J, Breckler L, et al: Atopic dermatitis in young children is associated with impaired interleukin-10 and interferon-gamma responses to allergens, vaccines and colonizing skin and gut bacteria. Clin Exp Allergy 2005;35:1309-1317.
Roessler A, Friedrich U, Vogelsang H, et al: The immune system in healthy adults and patients with atopic dermatitis seems to be affected differently by a probiotic intervention. Clin Exp Allergy 2008;38:93-102.

31 Prioult G, Nagler-Anderson C: Mucosal immunity and allergic responses: lack of regulation and/or lack of microbial stimulation? Immunol Rev 2005;206:204-218.

32 Smits HH, Engering A, van der KD, et al: Selective probiotic bacteria induce IL-10-producing regulatory $\mathrm{T}$ cells in vitro by modulating dendritic cell function through dendritic cell-specific intercellular adhesion molecule 3-grabbing nonintegrin. J Allergy Clin Immunol 2005;115:1260-1267.

33 Prescott SL, Wickens K, Westcott L, et al Supplementation with Lactobacillus rhamnosus or Bifidobacterium lactis probiotics in pregnancy increases cord blood interferongamma and breast milk transforming growth factor-beta and immunoglobin A detection. Clin Exp Allergy 2008;38:16061614.

34 Viljanen M, Pohjavuori E, Haahtela T, et al: Induction of inflammation as a possible mechanism of probiotic effect in atopic eczema-dermatitis syndrome. J Allergy Clin Immunol 2005;115:1254-1259.

35 Marschan E, Kuitunen M, Kukkonen K, et al: Probiotics in infancy induce protective immune profiles that are characteristic for chronic low-grade inflammation. Clin Exp Allergy 2008;38:611-618.
36 Prescott SL, Dunstan JA, Hale J, et al: Clinical effects of probiotics are associated with increased interferon-gamma responses in very young children with atopic dermatitis. Clin Exp Allergy 2005;35:1557-1564.

37 Snel J, Vissers YM, Smit BA, et al: Strain-specific immunomodulatory effects of Lactobacillus plantarum strains on birch-pollen-allergic subjects out of season. Clin Exp Allergy 2011;41:232-242.

38 van Wijk F, Wehrens EJ, Nierkens S, et al: CD4+CD25+ T cells regulate the intensity of hypersensitivity responses to peanut, but are not decisive in the induction of oral sensitization. Clin Exp Allergy 2007;37:572-581.

- 39 Wassenberg J, Nutten S, Audran R, et al: Effect of Lactobacillus paracasei ST11 on a nasal provocation test with grass pollen in allergic rhinitis. Clin Exp Allergy 2011;41: 565-573.

40 Lodinová-Zádníková R, Cukrowska B, Tlaskalova-Hogenova $\mathrm{H}$ : Oral administration of probiotic Escherichia coli after birth reduces frequency of allergies and repeated infections later in life (after 10 and 20 years). Int Arch Allergy Immunol 2003;131:209-211. 